\title{
Essential Amino Acids and Exercise Tolerance in Elderly Muscle-Depleted Subjects with Chronic Diseases: A Rehabilitation without Rehabilitation?
}

\author{
Roberto Aquilani, ${ }^{1}$ Giuseppe D’Antona, ${ }^{2,3}$ Paola Baiardi, ${ }^{4}$ Arianna Gambino, ${ }^{5}$ Paolo \\ Iadarola, ${ }^{6}$ Simona Viglio, ${ }^{3}$ Evasio Pasini, ${ }^{7}$ Manuela Verri, ${ }^{8}$ Annalisa Barbieri, ${ }^{9}$ and Federica \\ Boschi' $^{9}$ \\ ${ }^{1}$ Servizio di Fisiopatologia Metabolico-Nutrizionale e Nutrizione Clinica, Fondazione S. Maugeri, IRCCS, Istituto Scientifico di \\ Montescano, Via per Montescano 31, Montescano, 27040 Pavia, Italy \\ ${ }^{2}$ Laboratorio Universitario per lo Studio delle Attività Motorie nelle Malattie Rare (LUSAMMR), Via Ugo Foscolo 13, Voghera, 27058 \\ Pavia, Italy \\ ${ }^{3}$ Dipartimento di Medicina Molecolare, Università degli Studi di Pavia, Viale Taramelli 3/B, 27100 Pavia, Italy \\ ${ }^{4}$ Direzione Scientifica Centrale, Fondazione Salvatore Maugeri, IRCCS, Via Salvatore Maugeri 8-10, 27100 Pavia, Italy \\ ${ }^{5}$ Consorzio Valutazioni Biologiche e Farmacologiche, Via Luigi Porta 14, 27100 Pavia, Italy \\ ${ }^{6}$ Dipartimento di Biologia e Biotecnologie, Università degli Studi di Pavia, Via Ferrata 1, 27100 Pavia, Italy \\ ${ }^{7}$ Fondazione S. Maugeri, IRCCS, Istituto Scientifico di Lumezzane, Via Mazzini 9, Lumezzane, 25065 Brescia, Italy \\ ${ }^{8}$ Dipartimento di Medicina Legale, Scienze Forensi e Farmaco-Tossicologiche "A. Fornari", Sezione di Scienze Farmacologiche e \\ Tossicologiche, Università degli Studi di Pavia, 27100 Pavia, Italy \\ ${ }^{9}$ Dipartimento di Scienze del Farmaco, Università degli Studi di Pavia, Viale Taramelli 14, 27100 Pavia, Italy
}

Correspondence should be addressed to Federica Boschi; federica.boschi@unipv.it

Received 11 February 2014; Accepted 20 May 2014; Published 5 June 2014

Academic Editor: Enzo Nisoli

Copyright (C) 2014 Roberto Aquilani et al. This is an open access article distributed under the Creative Commons Attribution License, which permits unrestricted use, distribution, and reproduction in any medium, provided the original work is properly cited.

\begin{abstract}
Exercise intolerance remains problematic in subjects with chronic heart failure (CHF) and/or chronic obstructive pulmonary disease (COPD). Recent studies show that supplemented essential amino acids (EAAs) may exert beneficial effects on CHF/COPD physical capacity. The results from 3 investigations ( 2 conducted on CHF and 1 on COPD subjects) served as the basis for this paper. The 3 studies consistently showed that elderly CHF and COPD improved exercise intolerance after 1-3 months of EAA supplementation $(8 \mathrm{~g} / \mathrm{d}$ ). In CHF exercise capacity increased $18.7 \%$ to $23 \%$ (watts; bicycle test), and $12 \%$ to $22 \%$ (meters) in 6 min walking test. Moreover, patients reduced their resting plasma lactate levels (by 25\%) and improved tissue insulin sensitivity by $16 \%$ (HOMA index). COPD subjects enjoyed similar benefits as CHF ones. They increased physical autonomy by $78.6 \%$ steps/day and decreased resting plasma lactate concentrations by $23 \%$. EAA mechanisms explaining improved exercise intolerance could be increases in muscle aerobic metabolism, mass and function, and improvement of tissue insulin sensitivity (the latter only for the CHF population). These mechanisms could be accounted for by EAA's intrinsic physiological activity which increases myofibrils and mitochondria genesis in skeletal muscle and myocardium and glucose control. Supplemented EAAs can improve the physical autonomy of subjects with CHF/COPD.
\end{abstract}

\section{Introduction}

Patients with chronic heart failure $(\mathrm{CHF})$ and/or chronic obstructive pulmonary disease (COPD) often have reduced exercise tolerance, limiting participation in daily activities. Early onsets of fatigue and/or dyspnea are the symptoms responsible for this exercise intolerance. These symptoms are caused by peripheral mechanisms including abnormalities 
in skeletal muscle histology, metabolism, and function [1]. They are not caused by altered central cardiac function such as left ventricular ejection (CHF) [2] or the rate of airway obstruction (COPD) [3].

Exercise intolerance, accentuated in the elderly because of aging body changes, negatively impacts both functional and life prognosis. Indeed, it increases the risk of physical dependence, poor quality of life [4], and increased mortality rate both in CHF [5] and COPD [3]. In CHF, it is exercise intolerance and not heart function which is the most important prognostic factor [5], which furthermore is more accurate than hemodynamic/ventilator profiles at predicting outcomes [5]. Therefore, improved exercise intolerance of $\mathrm{CHF} / \mathrm{COPD}$ patients is a key for maintaining the subjects' autonomy and quality of life as well as for increasing survival.

At present, treatments to improve exercise intolerance in $\mathrm{CHF} / \mathrm{COPD}$ include specific pharmacological therapy and exercise training (ET). However, despite advanced pharmacological therapy, exercise intolerance still remains problematic in CHF/COPD individuals [4]. Although ET is the corner stone of cardiac and pulmonary rehabilitation (Rehab), it has not become an integral part of clinical management of patients with $\mathrm{CHF} / \mathrm{COPD}$ because only very few of these patients enter a Rehab programme [6] (in Europe less than $20 \%)$. The prevalence of COPD patients who undergo any Rehab protocol is not known. Moreover, not all subjects, including less elderly patients, can sustain ET because of compromised CHF/COPD and/or of more frequent comorbidities.

Recently, a nutritional approach has emerged as a potentially useful tool to improve exercise intolerance in $\mathrm{CHF} / \mathrm{COPD}$. Some studies [7-10] have found that chronic oral supplementation of essential amino acids (EAAs) $(8 \mathrm{~g} / \mathrm{d}$ over 1-3 months) significantly improves exercise intolerance of elderly patients with severe CHF/COPD, living at home or admitted to a Rehab setting.

In this paper we summarize the main findings of these studies and show that the EAA mechanisms leading to improved exercise intolerance rely on the intrinsic biochemical physiology of EAAs and on changes in skeletal muscle when EAAs are provided. We believe a better understanding of more comprehensive EAA activity can contribute to future clinical research. If confirmed by larger trials, EAA supplementation could allow elderly subjects with CHF/COPD, who are not on ET programmes, to achieve a good prognostic Rehab outcome.

\section{EAA-Induced Exercise Improvements in CHF/COPD and Their Mechanisms}

There is a huge literature on the effects of acute amino acid administration on exercise performance in healthy subjects and in athletes. However, only a few studies have been carried out on the chronic use of EAAs to improve exercise intolerance in elderly subjects suffering from severe CHF/COPD and altered body composition. Preliminary results show positive effects of EAAs on exercise intolerance. In Tables 1 and 2, the amino acid mixture used in these studies and their respectively changes in certain exercise variables after chronic
TABLE 1: Nutritional composition of an individual packet of supplementation, containing $4 \mathrm{~g}$ of an amino acid mixture, used in the clinical studies* reported in the current investigation [7-14].

\begin{tabular}{lc}
\hline Kcal & 35.3 \\
KJ & 149.9 \\
Total amino acids, of which & $4 \mathrm{~g}$ \\
L-Leucine & $1250 \mathrm{mg}$ \\
L-Lysine & $650 \mathrm{mg}$ \\
L-Isoleucine & $625 \mathrm{mg}$ \\
L-Valine & $625 \mathrm{mg}$ \\
L-Threonine & $350 \mathrm{mg}$ \\
L-Cysteine & $150 \mathrm{mg}$ \\
L-Histidine & $150 \mathrm{mg}$ \\
L-Phenylalanine & $100 \mathrm{mg}$ \\
L-Methionine & $50 \mathrm{mg}$ \\
L-Tyrosine & $30 \mathrm{mg}$ \\
L-Tryptophan & $20 \mathrm{mg}$ \\
\hline * Treated patients were given 2 packets daily (8g essential amino acids).
\end{tabular}

amino acid supplementation are reported, after evaluation from the original investigations [8-10].

2.1. EAAs and Exercise in CHF Subjects (Table 2). Three investigations reported that it is possible to increase exercise capacity of patients with CHF on maximal standard therapy. In one study [8] conducted on muscle-depleted patients (arm muscle area $<5^{\circ}$ percentile) $(n=44)$, two-month EAA treatment $(8 \mathrm{~g} / \mathrm{d})$ was associated with significant improvements in work performance. Six-minute walk distance (meters) increased by $22 \%$, being significantly more than their placebo counterparts $(+4 \%)$. The improvement in physical capacity was confirmed with the bicycle exercise test $(+18.7 \%$ watts versus $+3.5 \%$ watts in placebo). In this test, muscle aerobic metabolism, indicated by peak oxygen consumption $\left(\mathrm{mL} / \mathrm{min} / \mathrm{kg}\right.$; peak $\left.\mathrm{VO}_{2}\right)$, increased by $10.4 \%$ in EAA subjects and $0.08 \%$ in the placebo group. At rest, lactate concentration (micromole/L) decreased by $25 \%$ in EAAs whereas it worsened in placebo $\mathrm{CHF}(+15 \%)$ indicating a reduction for the EAA group but an increase in the placebo group of muscle anaerobic processes. Insulin resistance (HOMA index) diminished by $16 \%$ in treated $\mathrm{CHF}$, while it increased by $6.5 \%$ in the placebo group.

EAAs also improved nutritional status. Body weight increased in $80 \%$ of EAA subjects $(+3 \mathrm{Kg} ;+4.1 \%$ baseline $)$ and in $30 \%$ of placebo subjects $(+0.4 \mathrm{Kg} ;+0.6 \%)$. This difference in distribution between EAA and control groups was significant $(P<0.05)$. Skeletal muscle mass, indicated by arm muscle area, increased by $11.8 \%$ in EAA subjects and $8.4 \%$ in control (n.s.). Given that, before the protocol started, the criterium chosen for considering EAA efficacy was the combination of an increase in body weight $>1 \mathrm{Kg}$ and an increase of arm muscle area; the EAA associated changes in nutritional status were significantly higher in the treated compared to the placebo group. 
TABLE 2: Changes in exercise variables observed after EAA supplementation in subjects with chronic heart failure or chronic obstructive pulmonary disease.

\begin{tabular}{|c|c|c|c|c|}
\hline \multirow{2}{*}{ Exercise variables } & \multirow{2}{*}{ Disease } & \multirow{2}{*}{ Treatment duration } & \multicolumn{2}{|c|}{ Changes (\% pretreatment) } \\
\hline & & & Placebo & EAAs \\
\hline \multicolumn{5}{|l|}{ Mechanical work } \\
\hline \multirow{2}{*}{6 min WT (meters) } & $\mathrm{CHF}$ & 12 weeks & n.d. & $+12[9]$ \\
\hline & $\mathrm{CHF}$ & 8 weeks & +4 & $+22[8]$ \\
\hline \multirow{2}{*}{ Cycle ergometer (watts) } & $\mathrm{CHF}$ & 8 weeks & +3.5 & $+18.7[8]$ \\
\hline & $\mathrm{CHF}$ & 4 weeks & +4 & $+23[7]$ \\
\hline Steps (number/day) & COPD & 12 weeks & -7.8 & $+78.6[10]$ \\
\hline \multicolumn{5}{|l|}{ Metabolic variables during cycling } \\
\hline (a) Aerobic metabolism: & $\mathrm{CHF}$ & 8 weeks & & \\
\hline $\mathrm{VO}_{2}$ peak $(\mathrm{mL} / \mathrm{Kg} / \mathrm{min})$ & & & +0.08 & $+10.4[7,8]$ \\
\hline (b) Anaerobic metabolism: & $\mathrm{CHF}$ & 4 weeks & & \\
\hline \multicolumn{5}{|l|}{$\mathrm{VO}_{2}$ recovery time $(\mathrm{mL} / \mathrm{Kg} / \mathrm{min})$} \\
\hline (i) At $30 \%$ postpeak decline & & & -14 & $-58[7]$ \\
\hline (ii) At $50 \%$ postpeak decline & & & -1 & $-49[7]$ \\
\hline \multirow{2}{*}{ Resting plasma lactate $(\mu \mathrm{mol} / \mathrm{L})$} & $\mathrm{CHF}$ & 8 weeks & +15 & $-25[8]$ \\
\hline & COPD & 12 weeks & +13 & $-23[10]$ \\
\hline Resting insulin resistance (HOMA index) & $\mathrm{CHF}$ & 8 weeks & +6.5 & $-16[8]$ \\
\hline
\end{tabular}

WT: walking test; $\mathrm{VO}_{2}$ : oxygen uptake; $\mathrm{CHF}$ : chronic heart failure; COPD: chronic obstructive pulmonary disease; n.d.: not determined.

The benefit of EAAs for exercise tolerance was confirmed by two investigations performed in ambulatory CHF patients. In the first study [9], elderly CHF $(n=15)$ on 3-month EAA treatment $(8 \mathrm{~g} / \mathrm{d})$ improved their physical capacity at 6 min walk distance by $12 \%$ (the study had no placebo controls). In the second study [7], elderly CHF ( $n=$ 95) increased their exercise tolerance (bicycle exercise test) by $23 \%$ watts following 1-month EAA supplementation. In placebo subjects exercise capacity improved by $4 \%$. The postpeak $\mathrm{VO}_{2}$ recovery time, the length of which is an index of anaerobic metabolism, calculated at $30 \%$ postpeak $\mathrm{VO}_{2}$ decline decreased by $58 \%$ in EAA treated CHF and by $14 \%$ in placebo controls. At $50 \%$ postpeak $\mathrm{VO}_{2}$ decline, the $\mathrm{VO}_{2}$ recovery time decreased by $49 \%$ in EAA subjects and by only $1 \%$ in the placebo group.

To sum up, the available studies conducted on elderly subjects with CHF consistently reported significant improvements of exercise intolerance following chronic EAA supplementation. The plausible mechanisms included improved muscle aerobic metabolism energy-producing and nutritional status and reduced insulin resistance.

2.2. EAAs and Physical Capacity in COPD Subjects (Table 2). Similar results were also observed in ambulatory COPD population $(n=60)$ [10]. These patients were sarcopenic (bioimpedance analysis measure) and were on long-term oxygen therapy. Three-month EAA supplementation $(8 \mathrm{~g} / \mathrm{d})$ improved their physical capacity in terms of number of steps/day by $78.6 \%$ whereas subjects on placebo tended to have step diminution $(-7.8 \%)$. EAA treatment improved muscle efficiency in aerobic energy production as suggested by the $23 \%$ reduction in resting plasma lactate levels compared with the pre-EAA treatment period. In contrast, plasma lactate increased by $13 \%$ in the placebo group. It is interesting to note that both the time courses and the changes of plasma lactate concentrations were similar for muscle-depleted CHF [8] and sarcopenic COPD. This indirectly confirms the fact that the myopathy of CHF and COPD patients shares several metabolic alterations [1].

Again, similar to the CHF study, EAAs also improved patient nutritional status. Body weight increased by an average of $5.5 \mathrm{~kg}(+10.3 \%$ baseline $)$ of which $3.66 \mathrm{~kg}$ was fat free mass (FFM). In controls, body weight decreased by $3.5 \%$ but FFM increased by $0.6 \%(+3.8 \mathrm{~kg})$. Probably in this latter group, the overtime energy intake was inadequate and/or there was an increase of body water. Indeed, in treated subjects but not controls, physical capacity and FFM improvements were associated with increases in muscle strength (handgrip) $(+1.6 \mathrm{~kg} ;+7.4 \%)$, serum albumin concentrations $(+4.28 \mathrm{~g} / \mathrm{L})$, cognitive function $(+1.62$ scores at Minimental Test Examination), and quality of life perception (St. George's Respiratory Questionnaire, -2.7 scores). Like the CHF, improved muscle aerobic metabolism and nutritional status accounted for the improved exercise capacity of patients.

In addition, EAA treatment induced pluridistrict extramuscular anabolic activity in COPD subjects including that in the cerebral region, which is very sensitive to amino acid activity [27].

2.3. Other Interventional Studies Indirectly Suggest Improvement in Exercise Intolerance after EAA Supplementation. Given that muscle mass and, above all, muscle strength are good predictors of physical capacity, their increase after EAA supplementation $(8 \mathrm{~g} / \mathrm{d})$ indirectly suggests improved physical performance. 
Nutritional status significantly improved following EAA treatment (3 months, $8 \mathrm{~g} / \mathrm{d}$ ) in cachectic COPD (dual X-ray absorption measure) [11]. Patients' weight increased by $3.8 \pm$ $2.6 \mathrm{~kg}$ in the EAA group and $-0.1 \pm 1.1 \mathrm{~kg}$ in the placebo one. FFM increased in $69 \%$ and $15 \%$ of EAA and control patients, respectively. FFM average increase was $1.5 \pm 2.6 \mathrm{~kg}$ in EAA subjects and $-0.1 \pm 2.3 \mathrm{~kg}$ in controls.

In another study, EAA supplementation positively affected muscle strength in healthy elderly people [12] and in institutionalized old individuals [13]. In this study, EAAs subjects also significantly improved daily activity, mininutritional assessment score, depressive symptoms, and quality of life.

The effect of EAAs on insulin resistance [8] was confirmed in a cohort of elderly type 2 diabetics who showed long-term (60 weeks) better glucose control [14]. Interestingly, in another population of elderly diabetics, EAA supplementation improved cardiac performance as indicated by increased left ventricular ejection fraction [9]. This was also reported in healthy elderly individuals after 3 months of EAA treatment [12].

\section{EAA Mechanisms Leading to Improved Exercise Intolerance Rely on the Intrinsic Biochemical EAA Activities and Skeletal (Cardiac) Muscle Changes following Chronic EAA Treatment (Table 3)}

Improved muscle aerobic metabolism, prevalence of muscle anabolic processes, and reduction of insulin resistance (the latter in $\mathrm{CHF}$ ) are the main EAA mechanisms explaining increased exercise tolerance in both $\mathrm{CHF}$ and $\mathrm{COPD}$ subjects on maximal standard therapy [7-10]. These mechanisms mutually influence each other and, in turn, rely on the intrinsic biochemical EAA properties and profound skeletal (and cardiac) muscle changes following chronic EAA supplementation documented by a number of experimental studies. Here the mechanisms will be discussed separately for more clarity.

3.1. Improvements in Muscle Aerobic Energy Production (Table 3). Both CHF and CODP patients at rest are biochemically characterized by impaired formation of muscle energy availability (adenosine three phosphate-ATP and creatine phosphate-CP compounds) [1]. Adequate cell energy availability is essential to develop muscle strength and protein turnover (protein synthesis and proteolysis).

EAAs are the substrates which can enhance muscle aerobic metabolism because they act as fuel for Krebs cycle (tricarboxylic cycle acid: TCA), promoting mitochondria biogenesis and tackling the negative effects of insulin resistance on the TCA cycle. Indeed, EAAs can enter the TCA cycle at various levels and can be used as an alternative fuel for producing energy [28], as their activities are independent of insulin [21]. EAA supplements normalized the ATP content and production rate in aged rat gastrocnemius muscle similar to those of adult rats [15].
Furthermore, EAAs upregulate mitochondria synthesis and increase their volume and cellular density $[18,20]$. Interestingly, mitochondria biogenesis by EAAs is also upregulated in cardiac muscle [18]. In middle-aged mice, the essential branched-chain amino acid (BCAA) supplement increased mitochondrial biogenesis and sirtuin-1 expression (a member of sirtuin family linked to the life span extension), in cardiac and skeletal muscle, accompanied by enhanced physical performance [19].

All of these experimental studies suggest that chronic EAA supplementation both directly and indirectly increases muscle cell availability in high energy compounds indispensable for improving work performance and muscle strength in elderly individuals. This is independent of whether they are healthy [12] or affected by COPD [10] or CHF [9].

3.2. Improvements in Muscle Insulin Resistance (Table 3). EAA supplementation can make muscle aerobic metabolism more efficient by reducing insulin resistance [14]. EAAs directly upregulate insulin-receptor synthesis and its autophosphorylation [14]. Less insulin resistance diminishes the block of the cell pyruvate dehydrogenase complex caused by insulin resistance [8] and by circulating inflammatory cytokines in both CHF and COPD. Better activity of this enzymatic complex improves energy formation from glucose oxidation. Consequently, the lactate $\rightleftarrows$ pyruvate reaction shifts towards the right [8].

In induced diabetic rat hearts, long-term oral EAA supplementation increased mitochondrial cytochrome c oxidase and NADH-H activities and significantly shifted the ventricular myosin heavy chain pattern towards a faster phenotype [16]. Both in diabetic and in healthy mice, EAA supplements modulate the skeletal muscle redox state by improving the antioxidant defense system as shown by increased superoxide dismutase (SOD) expression and simultaneous decrease in heat shock proteins [17].

EAAs may reduce insulin resistance by lowering the circulating cytokine tumor necrosis factor alpha. This has been demonstrated in sarcopenic elderly subjects [14].

3.3. Improvements in Skeletal (and Cardiac) Muscle Anabolism. Improved nutritional status observed in muscledepleted patients $[8,10,11,13]$ clearly indicates that even subjects with severely reduced FFM and on maximal standard therapy can improve their nutritional status when supplemented with physiological amounts of EAAs. This is not surprising given that the physiological role of EAAs is both to increase protein synthesis and to decrease protein breakdown.

Chronic supplementation of essential BCAA leucine upregulates protein synthesis in skeletal muscle, adipose tissue, and liver [21] by enhancing activity synthesis of proteins involved in mRNA translation. Particularly relevant to elderly $\mathrm{CHF} / \mathrm{COPD}$ patients, leucine (and other EAAs) acts as a nutritional signaling molecule, quite independent of insulin [21]. However, amino acids regulate insulin signaling via mTOR nutrient signaling [29] and their adequate availability is indispensable for insulin to exert its anabolic activity [29]. On the other hand, reduced amino acid availability lowers 


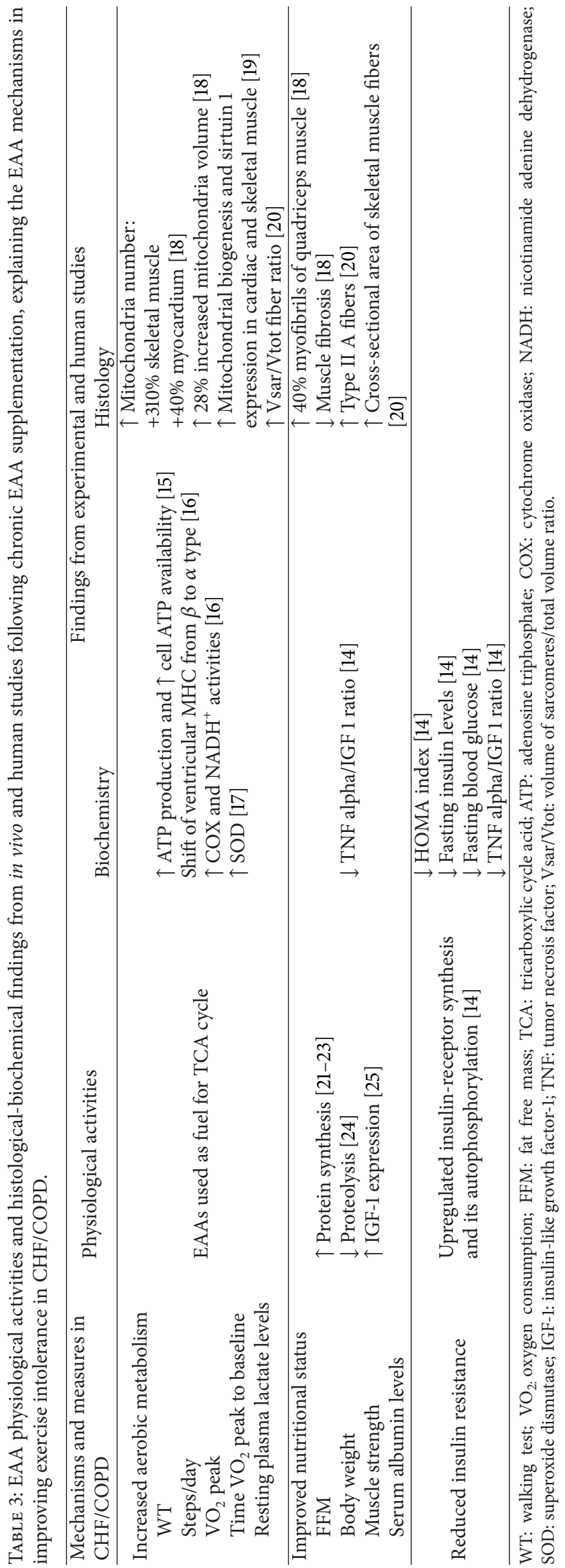


TABLE 4: Some similarities between the effects of exercise therapy and those following EAA supplementation.

\begin{tabular}{|c|c|c|}
\hline Measures & Exercise therapy $[1,5]$ & EAA supplementation \\
\hline \multicolumn{3}{|l|}{ Exercise capacity } \\
\hline Maximal oxygen uptake & Increased & Increased \\
\hline Six-minute walk distance & Increased & Increased \\
\hline Anaerobic threshold & Increased & Increased $^{*}$ \\
\hline Maximal incremental exercise duration & Increased & Increased \\
\hline Resting ejection fraction & Unchanged or slight increase & Slight, significant increase \\
\hline Resting lactate production & n.d. & Reduced \\
\hline \multicolumn{3}{|l|}{ Muscle structure and function } \\
\hline Muscle cross-sectional area & Increased & Increased $^{\wedge}$ \\
\hline Muscle fiber size & Increased & Increased $^{\wedge}$ \\
\hline Number of type I muscle fibers & Increased & Increased $^{\wedge}$ \\
\hline Mitochondrial numbers & Increased & Increased $^{\wedge}$ \\
\hline Mitochondrial cristae density & Increased & Increased $^{\wedge}$ \\
\hline Muscle dynamic strength & Increased & Increased $^{\wedge}$ \\
\hline Muscle fatigability & Reduced & Reduced $^{\wedge}$ \\
\hline
\end{tabular}

${ }^{*}$ Inferred from improved recovery time of maximal oxygen uptake to baseline value [26]; ${ }^{\wedge}$ in animals [15-20].

n.d.: not determined.

mTOR activation even with improved insulin signaling. This was documented in healthy and in insulin resistant human skeletal muscle [29]. Even small doses of exogenous EAAs can stimulate muscle protein metabolism [22].

Another pathway by which EAAs induce protein synthesis is by upregulating the hepatic production of the anabolic hormone insulin-like growth factor 1 (IGF-1). This hormone is dependent on EAA availability in the blood and acts only when there is adequate EAA availability. A study reported that $7.5 \mathrm{~g}$ EAAs (an amount very close to that used in the studies on EAAs and exercise intolerance) increased IGF-1 levels [25].

The fact that EAAs stimulate muscle protein metabolism in healthy elderly subjects [23] is of practical importance for elderly CHF/COPD individuals needing nutritional supplementation. This is because pharmaceutical formula reflecting the composition of a standard meal or high protein diet fails to increase muscle mass and strength as well as protein synthesis in the elderly [22]. EAA can induce net protein synthesis by reducing muscle protein breakdown. In human studies, the efficiency of protein use is due to the reduced sensitivity of proteolysis rather than any changes in protein synthesis [24].

Particularly interesting is the effect of leucine on protein metabolism in the myocardium. Leucine is the only amino acid that can inhibit protein degradation in the myocardium [30] and its inhibitory effect is mediated by extracellular leucine [30]. On the other hand, BCAA transamination in the heart is 3 times higher than in peripheral skeletal muscles [30]. The possible effect of EAAs on myocardium could explain the improved left ventricular dysfunction in healthy elderly individuals [9] and the faster exercise $\mathrm{VO}_{2}$ recovery time in elderly subjects with $\mathrm{CHF}[7,26]$.

In synthesis, experimental and human investigations have shown that chronic EAAs supplementation may increase and make the muscle aerobic energy production more efficient
TABLE 5: Amount of some types of high quality protein foods containing the same amount of essential amino acids $(8 \mathrm{~g})$ as the pharmaceutical formula used in the studies on exercise intolerance.

\begin{tabular}{lc}
\hline Food & $\mathrm{g}$ \\
\hline Lean beef meat & 97 \\
Chicken (breast) & 74 \\
Mortadella & 131 \\
Ham & 79 \\
Cheese (average of 6 types) & 105 \\
Canned tuna fish & 74 \\
Trout & 153 \\
Codfish & 97 \\
Eggs & 138 \\
Whole milk & 480 \\
\hline
\end{tabular}

and increase skeletal muscle mass/strength and mitochondria number and function. It has been reported [31] that $75 \%$ of the body's nitrogen requirement is supplied by leucine, isoleucine, valine, threonine, and lysine, all amino acids contained in the nutritional mixture used here. It is interesting to note that the mixture's amino acids were formulated in reciprocal stoichiometric ratios, specifically both to match metabolism energy needs and to maintain protein synthesis [31].

The histological and biochemical changes associated with EAA supplementation overlap those induced by ET Rehab (Table 4). It is also interesting to note that the EAA dose $(8 \mathrm{~g} / \mathrm{d})$ is the amount contained in higher protein quality foods usually consumed by both healthy and ill subjects (Table 5) [32].

To understand the importance of the reciprocal stoichiometric ratios of the amino acids in the mixture [31], very recently an experimental study [33] reported that excessive 
neurotransmitter serotonin derived from tryptophan and noradrenergic activity from tyrosine may negatively impact the physical activity status particularly in sedentary animals. Moreover excessive tryptophan consumption can reduce physical activity by inducing central fatigue [34]. Interestingly, excessive tryptophan may also limit the blood-brainbarrier passage of leucine, isoleucine, valine, and tyrosine, therefore reducing their availability within the brain structure [35] and contributing to further limiting physical capacity.

\section{Conclusions}

Elderly patients with $\mathrm{CHF} / \mathrm{COPD}$ when supplemented with EAAs not only may improve exercise intolerance but also can achieve some prognostic outcomes typical of ET Rehab. Obviously, EAAs does not substitute comprehensive Rehab but may be very useful for elderly subjects with CHF/COPD who, for various reasons, cannot undergo ET Rehab.

\section{Conflict of Interests}

The authors have no conflict of interests regarding the publication of this paper.

\section{Acknowledgment}

The authors would like to thank Professor Robert Coates (Centro Linguistico, Università Bocconi, Milano, Italy), medical writer, for his linguistic revision.

\section{References}

[1] H. R. Gosker, E. F. M. Wouters, G. J. van der Vusse, and A. M. W. J. Schols, "Skeletal muscle dysfunction in chronic obstructive pulmonary disease and chronic heart failure: underlying mechanisms and therapy perspectives," The American Journal of Clinical Nutrition, vol. 71, no. 5, pp. 1033-1047, 2000.

[2] A. J. S. Coats, "Optimizing exercise training for subgroups of patients with chronic heart failure," European Heart Journal, vol. 19, supplement, pp. O29-O34, 1998.

[3] B. Waschki, A. Kirsten, O. Holz et al., "Physical activity is the strongest predictor of all-cause mortality in patients with COPD: a prospective cohort study," Chest, vol. 140, no. 2, pp. 331-342, 2011.

[4] I. Hagerman, R. Tyni-Lenné, and A. Gordon, "Outcome of exercise training on the long-term burden of hospitalisation in patients with chronic heart failure. A restrospective study," International Journal of Cardiology, vol. 98, no. 3, pp. 487-491, 2005.

[5] M. D. Witham, A. D. Struthers, and M. E. T. McMurdo, "Exercise training as a therapy for chronic heart failure: can older people benefit?" Journal of the American Geriatrics Society, vol. 51, no. 5, pp. 699-709, 2003.

[6] M. F. Piepoli, V. Conraads, U. Corrà et al., "Exercise training in heart failure: from theory to practice. A consensus document of the Heart Failure Association and the European Association for Cardiovascular Prevention and Rehabilitation," European Journal of Heart Failure, vol. 13, no. 4, pp. 347-357, 2011.

[7] R. Aquilani, S. Viglio, P. Iadarola et al., "Oral amino acid supplements improve exercise capacities in elderly patients with chronic heart failure," American Journal of Cardiology, vol. 101, no. 11, supplement, pp. S104-S110, 2008.

[8] R. Aquilani, C. Opasich, A. Gualco et al., "Adequate energyprotein intake is not enough to improve nutritional and metabolic status in muscle-depleted patients with chronic heart failure," European Journal of Heart Failure, vol. 10, no. 11, pp. 1127-1135, 2008.

[9] R. Scognamiglio, A. Testa, R. Aquilani, F. S. Dioguardi, and E. Pasini, "Impairment in walking capacity and myocardial function in the elderly: is there a role for nonpharmacologic therapy with nutritional amino acid supplements?" American Journal of Cardiology, vol. 101, no. 11, supplement, pp. S78-S81, 2008.

[10] R. W. Dal Negro, A. Testa, R. Aquilani et al., "Essential amino acid supplementation in patients with severe COPD: a step towards home rehabilitation," Monaldi Archives for Chest Disease, vol. 77, no. 2, pp. 67-75, 2012.

[11] S. Baldi, R. Aquilani, G. D. Pinna, P. Poggi, A. de Martini, and C. Bruschi, "Fat-free mass change after nutritional rehabilitation in weight losing COPD: role of insulin, C-reactive protein and tissue hypoxia," International Journal of Chronic Obstructive Pulmonary Disease, vol. 5, no. 1, pp. 29-39, 2010.

[12] R. Scognamiglio, R. Piccolotto, C. Negut, A. Tiengo, and A. Avogaro, "Oral amino acids in elderly subjects: effect on myocardial function and walking capacity," Gerontology, vol. 51, no. 5, pp. 302-308, 2005.

[13] M. Rondanelli, A. Opizzi, N. Antoniello et al., "Effect of essential amino acid supplementation on quality of life, Amino acid profile and strength in institutionalized elderly patients," Clinical Nutrition, vol. 30, no. 5, pp. 571-577, 2011.

[14] S. B. Solerte, M. Fioravanti, E. Locatelli et al., "Improvement of blood glucose control and insulin sensitivity during a longterm (60 weeks) randomized study with amino acid dietary supplements in elderly subjects with type 2 diabetes mellitus," American Journal of Cardiology, vol. 101, no. 11, supplement, pp. S82-S88, 2008.

[15] C. Chen Scarabelli, R. B. McCauley, Z. Yuan et al., "Oral administration of amino acidic supplements improves protein and energy profiles in skeletal muscle of aged rats: elongation of functional performance and acceleration of mitochondrial recovery in adenosine triphosphate after exhaustive exertion," American Journal of Cardiology, vol. 101, no. 11, supplement, pp. S42-S48, 2008.

[16] M. A. Pellegrino, C. Patrini, E. Pasini et al., "Amino acid supplementation counteracts metabolic and functional damage in the diabetic rat heart," American Journal of Cardiology, vol. 101, no. 11, supplement, pp. S49-S56, 2008.

[17] L. Brocca, G. D'Antona, A. Bachi, and M. A. Pellegrino, "Amino acid supplements improve native antioxidant enzyme expression in the skeletal muscle of diabetic mice," American Journal of Cardiology, vol. 101, no. 11, supplement, pp. S57-S62, 2008.

[18] G. Corsetti, E. Pasini, G. D'Antona et al., "Morphometric changes induced by amino acid supplementation in skeletal and cardiac muscles of old mice," American Journal of Cardiology, vol. 101, no. 11, supplement, pp. S26-S34, 2008.

[19] G. D'Antona, M. Ragni, A. Cardile et al., "Branched-chain amino acid supplementation promotes survival and supports cardiac and skeletal muscle mitochondrial biogenesis in middle-aged mice," Cell Metabolism, vol. 12, no. 4, pp. 362-372, 2010. 
[20] O. Pansarasa, V. Flati, G. Corsetti, L. Brocca, E. Pasini, and G. D'Antona, "Oral amino acid supplementation counteracts age-induced sarcopenia in elderly rats," American Journal of Cardiology, vol. 101, no. 11, supplement, pp. S35-S41, 2008.

[21] J. C. Anthony, T. G. Anthony, and D. K. Layman, "Leucine supplementation enhances skeletal muscle recovery in rats following exercise," Journal of Nutrition, vol. 129, no. 6, pp. 11021106, 1999.

[22] E. Volpi, H. Kobayashi, M. Sheffield-Moore, B. Mittendorfer, and R. R. Wolfe, "Essential amino acids are primarily responsible for the amino acid stimulation of muscle protein anabolism in healthy elderly adults," The American Journal of Clinical Nutrition, vol. 78, no. 2, pp. 250-258, 2003.

[23] D. Paddon-Jones, K. R. Short, W. W. Campbell, E. Volpi, and R. R. Wolfe, "Role of dietary protein in the sarcopenia of aging," The American Journal of Clinical Nutrition, vol. 87, no. 5, pp. 1562S-1566S, 2008.

[24] M. Kadowaki and T. Kanazawa, "Amino acids as regulators of proteolysis," Journal of Nutrition, vol. 133, no. 6, supplement 1 , pp. 2052S-2056S, 2003.

[25] E. L. Dillon, M. Sheffield-Moore, D. Paddon-Jones et al., "Amino acid supplementation increases lean body mass, basal muscle protein synthesis, and insulin-like growth factor-I expression in older women," Journal of Clinical Endocrinology and Metabolism, vol. 94, no. 5, pp. 1630-1637, 2009.

[26] A. Cohen-Solal, T. Laperche, D. Morvan, M. Geneves, B. Caviezel, and R. Gourgon, "Prolonged kinetics of recovery of oxygen consumption after maximal graded exercise in patients with chronic heart failure: analysis with gas exchange measurements and NMR spectroscopy," Circulation, vol. 91, no. 12, pp. 2924-2932, 1995.

[27] R. Aquilani, M. Boselli, F. Boschi et al., "Branched-chain amino acids may improve recovery from a vegetative or minimally conscious state in patients with traumatic brain injury: a pilot study," Archives of Physical Medicine and Rehabilitation, vol. 89, no. 9, pp. 1642-1647, 2008.

[28] T. Kutsuzawa, S. Shioya, D. Kurita, and M. Haida, "Plasma branched-chain amino acid levels and muscle energy metabolism in patients with chronic obstructive pulmonary disease," Clinical Nutrition, vol. 28, no. 2, pp. 203-208, 2009.

[29] M. J. Drummond, J. A. Bell, S. Fujita et al., "Amino acids are necessary for the insulin-induced activation of mTOR/S6K1 signaling and protein synthesis in healthy and insulin resistant human skeletal muscle," Clinical Nutrition, vol. 27, no. 3, pp. 447-456, 2008.

[30] B. H. L. Chua, "Specificity of leucine effect on protein degradation in perfused rat heart," Journal of Molecular and Cellular Cardiology, vol. 26, no. 6, pp. 743-751, 1994.

[31] F. S. Dioguardi, "Wasting and the substrate-to-energy controlled pathway: a role for insulin resistance and amino acids," American Journal of Cardiology, vol. 93, supplement 1, no. 8, pp. 6-12, 2004.

[32] E. Carnovale, L. Marletta, and Istituto Nazionale di Ricerca per Gli Alimenti e la Nutrizione, Tabelle di Composizione Degli Alimenti, Istituto Superiore Nazionale della Nutrizione, Roma, Italy, 1989.

[33] M. Fleshner, B. N. Greenwood, and R. Yirmiya, "Neuronalglial mechanisms of exercise-evoked stress robustness," Current Topics in Behavioral Neurosciences, 2014.

[34] T. Yamamoto, H. Azechi, and M. Board, "Essential role of excessive tryptophan and its neurometabolites in fatigue," The
Canadian Journal of Neurological Sciences, vol. 39, no. 1, pp. 4047, 2012.

[35] J. D. Fernstrom, "Large neutral amino acids: dietary effects on brain neurochemistry and function," Amino Acids, vol. 45, no. 3, pp. 419-430, 2013. 

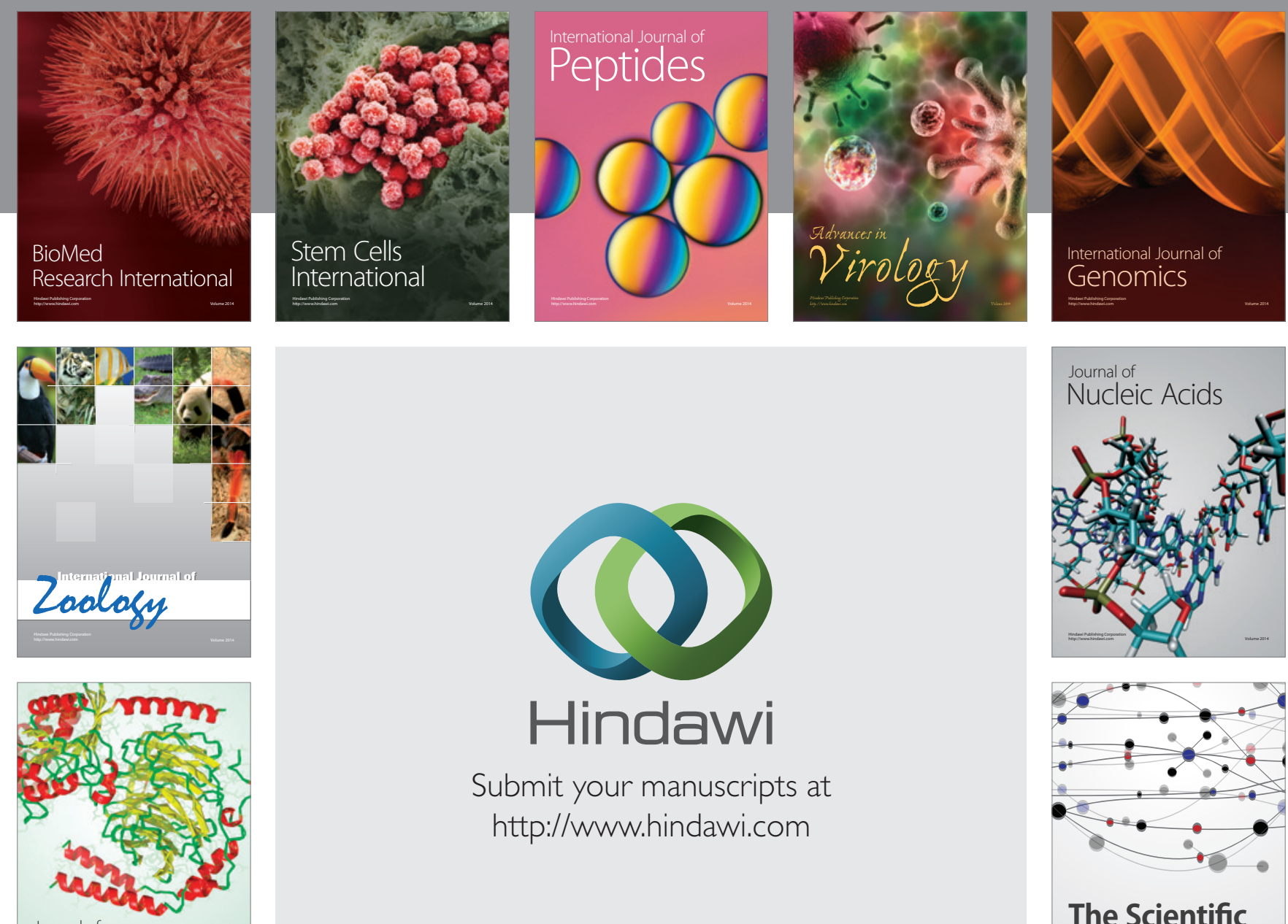

Submit your manuscripts at

http://www.hindawi.com

Journal of
Signal Transduction
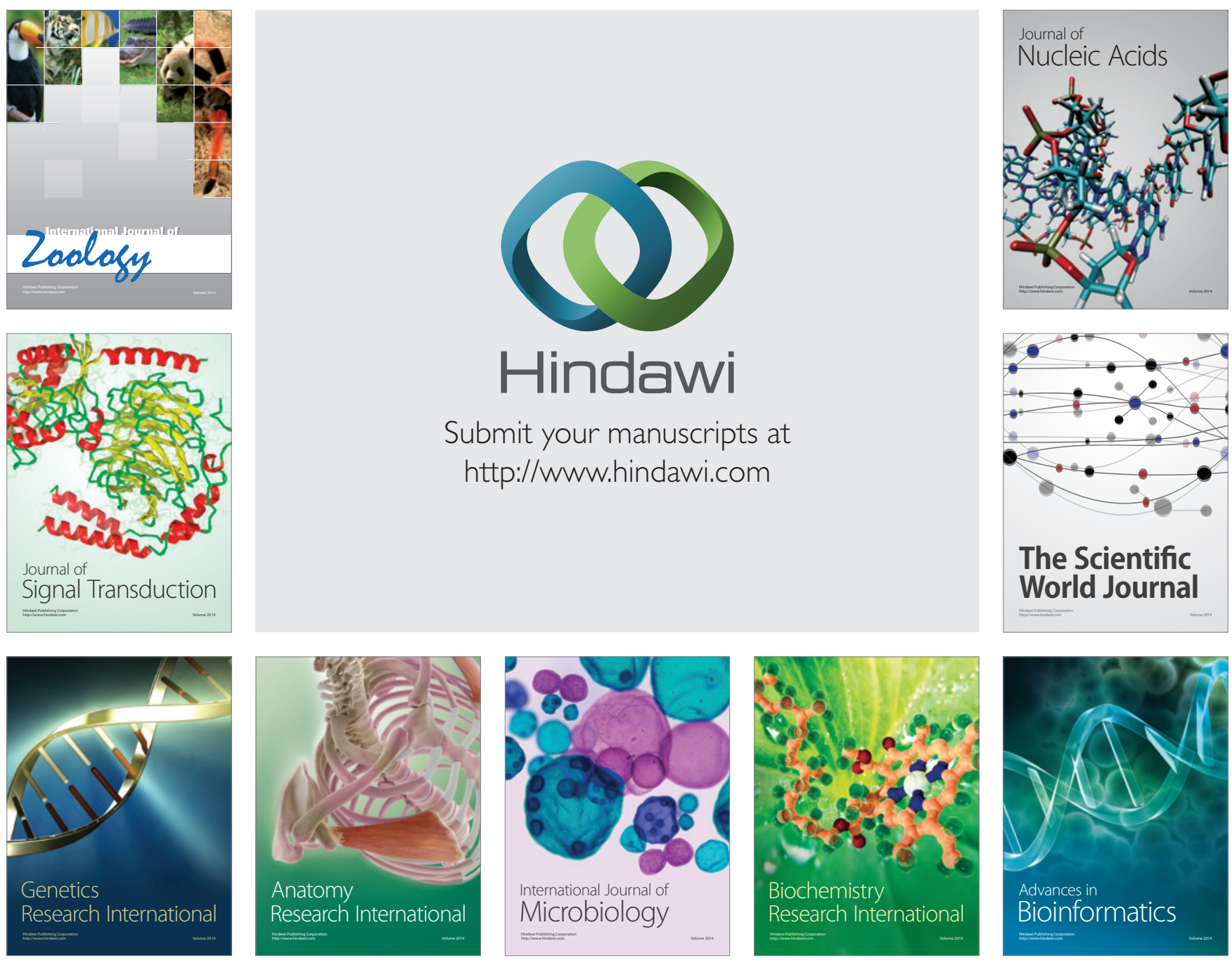

The Scientific World Journal
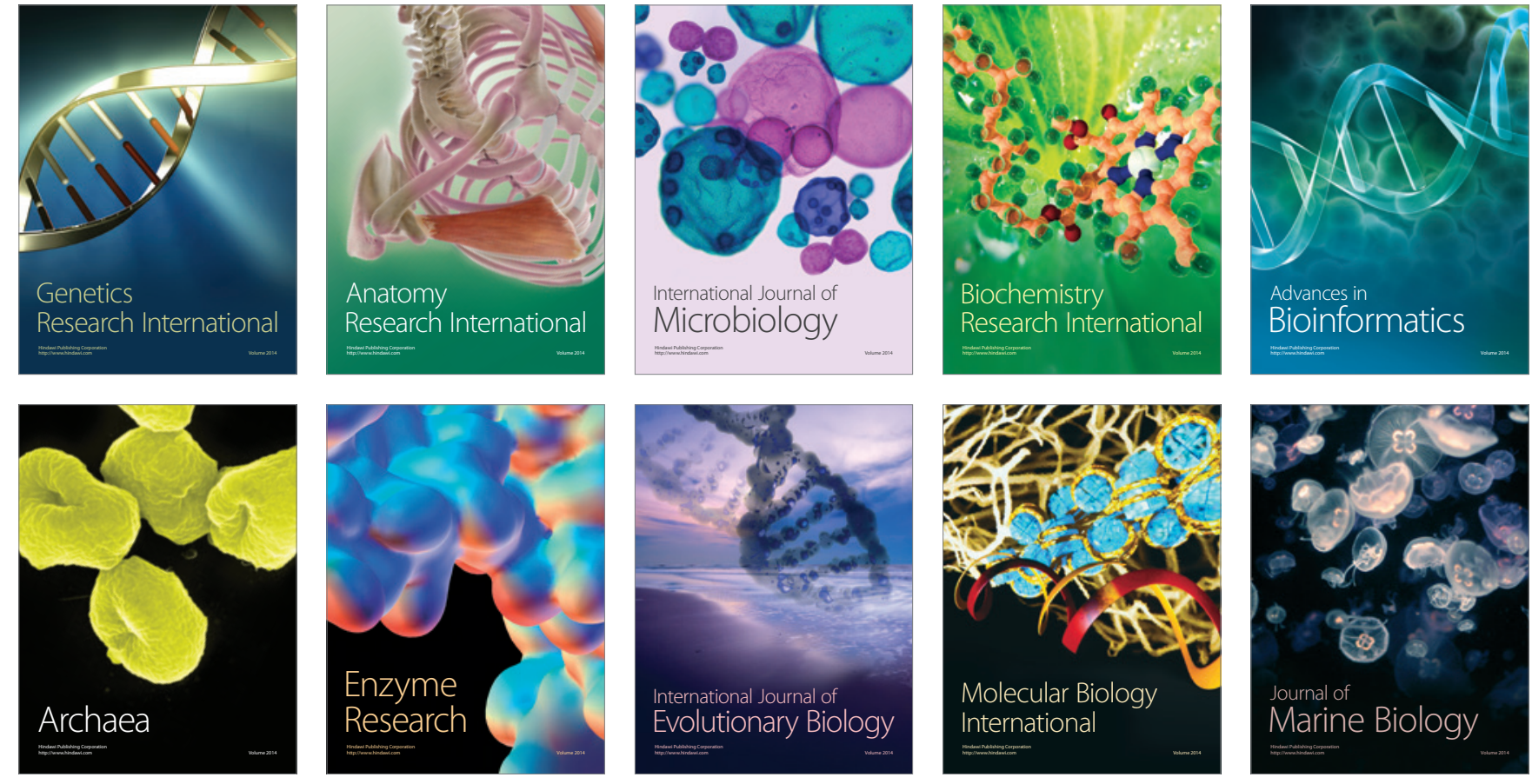